\title{
TECNOLOGIA MÓVEL PARA REGISTROS DA AVALIAÇÃO CLÍNICA DE RECÉM- NASCIDOS*
}

\author{
Laura Cristhiane Mendonça Rezende ${ }^{1}$, Sérgio Ribeiro dos Santos ${ }^{2}$, Luciana de Medeiros Lima ${ }^{3}$, Allana de \\ Oliveira Albuquerque Padilha ${ }^{4}$, Mouryene de Andrade Moreira Palmeira ${ }^{5}$, Cleidjane Gomes Faustino ${ }^{5}$
}

\begin{abstract}
RESUMO: Objetivou-se conhecer a opinião de enfermeiros sobre a utilização da tecnologia móvel para o registro da avaliação clínica de recém-nascidos. Estudo de natureza descritiva e exploratória com abordagem qualitativa, desenvolvido na Unidade de Terapia Intensiva Neonatal de um hospital do município de João Pessoa, estado da Paraíba. Os dados foram coletados no período de setembro a outubro de 2014, com entrevista semiestruturada e posterior análise de conteúdo. Foi possível identificar três categorias que destacaram a opinião das participantes: Categoria 1 - Reconhecendo a importância da tecnologia móvel para o registro de enfermagem; Categoria 2 Sentindo algumas dificuldades de manusear a tecnologia; Categoria 3-Apontando as contribuições do tablet para o processo de trabalho do enfermeiro. Conclui-se que a tecnologia móvel flexibiliza a realização das atividades de registro pelo enfermeiro, além de otimizar o tempo dispensado por estes profissionais nas atividades de documentação.
\end{abstract}

DESCRITORES: Tecnologia em saúde; Processamento eletrônico de dados; Registros de enfermagem.

\section{MOBILE TECHNOLOGY FOR RECORDING THE CLINICAL ASSESSMENT OF NEWBORNS}

ABSTRACT: The objective of this study was to learn the opinion of nurses on the use of mobile technology for the registration of newborns' clinical assessment. A descriptive and exploratory study, with a qualitative approach, was developed in the Neonatal Intensive Care Unit of a hospital in the city of João Pessoa, state of Paraíba, Brazil. Data were collected from September to October 2014 with semi-structured interviews and subsequent content analysis. It was possible to identify three categories that highlighted the opinion of participants: Category 1 - Recognition of the importance of mobile technology for nursing records; Category 2 Experiencing difficulties in handling the technology; Category 3 - Pointing out the tablet contributions to nursing work. In conclusion, mobile technology eases the activities of registration by nurses in addition to optimizing the time spent by these professionals in the documentation process.

DESCRIPTORS: Biomedical Technology; Automatic Data Processing; Nursing Records.

\section{TECNOLOGÍA MÓVIL PARA REGISTROS DE LA EVALUACIÓN CLÍNICA DE RECIÉN NACIDOS}

RESUMEN: El objetivo fue conocer la opinión de enfermeros sobre el uso de la tecnología móvil para el registro de la evaluación clínica de los recién nacidos. El estudio, descriptivo y exploratorio con enfoque cualitativo, fue desarrollado en la Unidad de Cuidados Intensivos Neonatales de un hospital en la ciudad de João Pessoa, Estado de Paraiba, Brasil. Los datos fueron obtenidos desde septiembre hasta octubre de 2014, con entrevistas semi estructuradas y posterior análisisdel contenido.

Fue posible identificar tres categorías de relieve a partir de la opinión de los participantes: Categoría 1 - El reconocimiento sobre la importancia de la tecnología móvil para los registros de enfermería; Categoría 2 - La percepción de algunas dificultades para el manejo de la tecnología; Categoría 3 -La señalización con respecto a las contribuciones de la tableta (tablet)al trabajo de enfermería. Se llega a la conclusión de que la tecnología móvil facilita la realización delregistro hecho por el enfermero, además deoptimizar el tiempo de los profesionales en el proceso de documentación de la información.

DESCRIPTORES: Tecnología en salud; Procesamiento electrónico de datos; Registros de enfermería.

*Artigo extraído da dissertação intitulada: "Sistematização da Assistência de Enfermagem em Unidade de Terapia Intensiva Neonatal: desenvolvimento de um protótipo para utilização em dispositivos móveis." Universidade Federal da Paraíba, 2015.

${ }^{1}$ Enfermeira. Mestre em Enfermagem. Docente da Faculdade Maurício de Nassau. João Pessoa, PB, Brasil.

${ }^{2}$ Enfermeiro. Doutor em Sociologia. Docente do Departamento de Enfermagem Clínica da Universidade Federal da Paraíba. João Pessoa, PB, Brasil.

${ }^{3}$ Enfermeira. Mestre em Enfermagem. Docente da Faculdade de Enfermagem São Vicente de Paula. João Pessoa, PB, Brasil.

${ }^{4}$ Enfermeira. Especialista em Enfermagem e Terapia Intensiva. Hospital Municipal Santa Isabel. João Pessoa, PB, Brasil.

${ }^{5}$ Enfermeira. Faculdade Internacional da Paraíba. João Pessoa, PB, Brasil.

Faculdade Maurício de Nassau

R. Josué Guedes Pereira, 221 - 58035-040 - João Pessoa, PB, Brasil

E-mail: lauracristhiane@hotmail.com 


\section{- INTRODUÇÃO}

O acelerado processo de modernização científica e tecnológica tem gerado novas formas de construir o conhecimento e de se estabelecer relações com o mundo do trabalho. Acredita-se que, nos próximos anos, os avanços da tecnologia computacional irão revolucionar os processos em todos os níveis dos serviços de enfermagem em instituições de saúde e proporcionar benefícios operacionais e estratégicos para a organização e o desenvolvimento da prática profissional ${ }^{(1)}$.

Nesse contexto, as tecnologias de informação móveis e sem fio encontram-se entre os principais temas discutidos atualmente, tanto no meio organizacional quanto no acadêmico. A crescente aplicação dessas tecnologias faz emergir uma série de questões relativas à sua criação, escolha, adaptação e vantagens de utilização, principalmente no âmbito da saúde ${ }^{(2)}$.

No Brasil e no mundo, encontram-se diferentes serviços que são realizados com o auxílio da tecnologia móvel, dentre eles os serviços de saúde. Nesta área, estes dispositivos contribuem, por exemplo, para a recepção em pronto-socorro, o encurtamento da fila de espera, processamento de dados clínicos e até o completo serviço móvel com laptops do tipo "tablet" associados a Personal Assistant Digital (PDAs) e a celulares ${ }^{(3)}$.

Dentre os dispositivos móveis utilizados na atualidade, os que se destacam são os tablets, equipamentos considerados vantajosos devido ao seu tamanho reduzido, facilidade de uso, grande capacidade de armazenamento, conectividade e alto poder de processamento. Demonstram ser instrumentos poderosos em atividades que exigem mobilidade, como por exemplo, o registro da etapa de coleta de dados para a sistematização da assistência de enfermagem ${ }^{(4-5)}$.

Um estudo realizado com enfermeiros de uma unidade de terapia semi-intensiva de um hospital escola do interior do estado de São Paulo apontou que uma das dificuldades dos enfermeiros no emprego de outras ferramentas computacionais, que não os dispositivos móveis, consistia no transporte das informações coletadas junto do paciente para o microcomputador. Essa dificuldade tinha relação com a distância entre o hardware e o leito do paciente, onde o enfermeiro registrava em papel a coleta de dados acerca de um paciente e, posteriormente, procedia à transcrição para a máquina. Esse é um dos principais problemas ao utilizar computadores fixos para registrar a prática de enfermagem, uma vez que a atividade assistencial envolve mobilidade dos enfermeiros para o atendimento de diversos pacientes internados em unidade hospitalar ${ }^{(6)}$.

Com o dispositivo móvelà mão, é possível acessar, coletar e documentar informações sobre o paciente em seu próprio leito, realizar etapas do processo de enfermagem e acompanhar a necessidade de mobilidade dos profissionais nas ações de assistência ao paciente. É possível, também, reduzir o tempo despendido na documentação das atividades e diminuir a probabilidade de perda das informações que passam a ser armazenadas no próprio dispositivo e não em papéis, o que demonstra como as características de flexibilidade e dinamismo convergem entre si e contribuem para a produtividade da assistência de enfermagem ${ }^{(7)}$.

Diante desta realidade, a computação móvel emerge como uma tecnologia inovadora para a assistência de enfermagem, por meio de sua aplicação via dispositivo móvel a outros computadores e da interface de redes sem fio integrada e planejada. A utilização paralela da computação móvel e o acesso a esse tipo de rede poderão, sem dúvida, auxiliar consideravelmente o dia a dia enfermeiros ${ }^{(8)}$.

Apesar das inúmeras vantagens obtidas por meio da sua utilização, a aplicação dos recursos tecnológicos na saúde e na Enfermagem ainda é dificultada, devido à falta de ações efetivas, uma vez que muitos gestores não compreendem a relação dinâmica de elementos relativos à estrutura, ao processo e à obtenção de resultados, frequentemente comprometidos por condições de trabalho, em que a manutenção de tecnologia necessária ainda é um sério entrave ${ }^{(9)}$.

Considerando o exposto, o estudo teve como objetivo conhecer a opinião de enfermeiros sobre a utilização de um tablet para registros da avaliação clínica de recém-nascidos em uma Unidade de Terapia Intensiva Neonatal. 


\section{- MÉTODO}

Trata-se de um estudo de natureza descritiva e exploratória com abordagem qualitativa, realizado na Unidade de Terapia Intensiva Neonatal (UTIN) de um hospital escola, localizada no município de João Pessoa, estado da Paraíba. A referida unidade é responsável pelo cuidado de recém-nascidos $(0$ a 28 dias de vida) que apresentem distúrbios clínicos e/ou cirúrgicos, e possui uma equipe de enfermagem composta por seis enfermeiras e nove técnicos de enfermagem.

Para verificar a opinião de enfermeiras sobre o uso dos dispositivos móveis nas atividades de registro, utilizou-se um protótipo de software com um banco de dados para avaliação clínica de recémnascidos, desenvolvido a partir das informações utilizadas nos formulários e livros de enfermagem da unidade. Para as enfermeiras da UTIN manusearem o sistema, foi utilizado um dispositivo móvel do tipo tablet, 7", dual core, Android 4.0, conectado com a rede wifi da unidade, que permaneceu durante todo o período do estudo na unidade, em todos os turnos de atividades.

Durante a primeira semana do estudo, foi realizado um treinamento. Inicialmente foi apresentado o sistema às profissionais, explicado seu funcionamento e sugeridos os primeiros contatos com a tecnologia móvel. No acompanhamento da utilização pelas enfermeiras, em pelo menos dois turnos das 24 horas do serviço de Enfermagem na UTIN, a pesquisadora e colaboradores treinados revezaramse para acompanhar a utilização do sistema, tirar dúvidas, se fosse necessário fazer observações.

Após o período de um mês de manuseio do dispositivo móvel, foram realizadas entrevistas com cinco enfermeiras. Os dados foram coletados no período de setembro a outubro de 2014, por meio de um roteiro semiestruturado, que continha questões para identificar a opinião das participantes quanto à importância de se trabalhar com a tecnologia móvel na avaliação clínica dos recém-nascidos; as dificuldades em manusear o tablet; e as principais contribuições do dispositivo para a prática do enfermeiro. Os dados foram analisados através do método de análise temática de conteúdo de $\operatorname{Bardin}^{(10)}$.

Como critérios de inclusão foram adotados: ter vínculo com o hospital, atuar na UTIN há pelo menos um ano, ter participado do treinamento e ter utilizado o software pelo menos uma vez a cada plantão. Das seis enfermeiras que compõem o quadro de pessoal da unidade, apenas uma não atendeu aos critérios, tendo em vista que se encontrava em licença maternidade.

Quanto aos aspectos éticos, obedeceu-se às orientações inerentes ao protocolo de pesquisa contido na Resolução $\mathrm{n}^{\circ} 466 / 12 \mathrm{CNS}^{(11)}$. Antes do início da pesquisa, o projeto foi encaminhado ao Comitê de Ética em Pesquisa (CEP), tendo sido aprovado e registrado no Sistema Nacional de Informação sobre Ética em Pesquisa envolvendo Seres Humanos (SISNEP) com CAAE-25890914.5.0000.5183.

É importante ressaltar que os participantes não receberam benefícios nem correram risco de discriminação ao participar da pesquisa. Além disso, foram comunicados de que poderiam se recusar a participar da pesquisa ou responder a qualquer pergunta relacionada a ela. Para assegurar a confidencialidade das informações e proteger a identidade dos colaboradores, eles foram identificados com a letra "E", adicionada dos números de 1 a 5.

\section{RESULTADOS}

Quanto à caracterização das participantes do estudo, foi verificado que o tempo de formação variou de 10 a 30 anos e que todas tinham algum tipo de especialização - em educação, saúde coletiva, saúde do trabalhador e enfermagem pediátrica; apenas uma enfermeira com o título de Mestre em Enfermagem. O tempo de atuação profissional na unidade oscilou entre 10 e 12 anos.

A partir da compreensão das respostas dos questionamentos sobre a utilização do tablet, foi possível identificar três categorias temáticas: Categoria 1 - Reconhecendo a importância da tecnologia móvel para o registro de enfermagem; Categoria 2 - Sentindo algumas dificuldades em manusear a tecnologia; e Categoria 3 - Apontando as contribuições do tablet para o processo de trabalho do enfermeiro. 


\section{Categoria 1 - Reconhecendo a importância da tecnologia móvel para o registro de enfermagem}

Nesta categoria, buscou-se compreender a opinião das enfermeiras quanto à importância do dispositivo móvel para a Unidade de Terapia Intensiva Neonatal em que o estudo foi realizado. Algumas enfermeiras disseram que uma das principais vantagens do sistema para tablet foi a independência de utilização do único dispositivo fixo presente na unidade, como observado nas falas a seguir:

Na UTI temos somente um dispositivo fixo, que o profissional médico já ocupa por um longo período de tempo, e a gente fica naquela dependência e é muito ruim, o móvel te dá mais liberdade. (E1)

O computador é do profissional médico praticamente a manhã inteira. Um só computador dificulta bastante, se a gente tem o tablet, o sistema móvel, eu já pego já sento em outro lugar. (E3)

Observa-se, portanto, que a unidade não dispõe de recursos tecnológicos suficientes para que os profissionais de saúde possam desempenhar as atividades de registro, exigindo que as enfermeiras protelassem esta documentação para outro momento. Foi destacado que com o dispositivo móvel este problema passava a ser superado.

\section{Categoria 2 - Sentindo algumas dificuldades de manusear a tecnologia}

A segunda categoria objetivou identificar as dificuldades encontradas pelas enfermeiras ao utilizarem o dispositivo móvel para registrar as informações referentes ao cuidado de enfermagem. Seguem alguns depoimentos que mostram opiniões distintas a este respeito:

Consegui avançar, muito lentamente, mas consegui. Dificuldade de acesso mesmo. Eu acho que dificuldades a gente tem, tá no começo, de adaptação. (E1)

Sim, tive dificuldades. Na verdade, eu nunca mexi num tablet, mas depois que me explicaram eu achei fácil. (E2)

Só tive dificuldade de ligar quando ele descarregou. (E3)

Em contrapartida, algumas enfermeiras não apresentaram dificuldades para manusear a tecnologia móvel:

Não, dificuldade mesmo eu não encontrei não. Tá tranquilo. Se tivesse a possibilidade de ficar com uma máquina com a gente, um tablet... eu acho que dariam certo os registros. (E3)

Na verdade no manuseio do tablet não houve problema. (E4)

Os relatos demonstram que algumas enfermeiras apresentaram dificuldades em utilizar o tablet (E1; E2), as quais estão relacionadas à falta de familiaridade/experiência das profissionais com a tecnologia e ao fato de nunca terem usado esse tipo de tecnologia móvel em seu processo de trabalho (E2). Isso explica a dificuldade de utilizar o dispositivo, principalmente, por ser do tipo touchscreen.

\section{Categoria 3 - Apontando as contribuições do tablet para o processo de trabalho do enfermeiro}

$\mathrm{Na}$ terceira categoria, foi visto que as enfermeiras reconhecem as contribuições do dispositivo móvel com o qual se possam registrar dados clínicos e da assistência de enfermagem. Foi possível também observar as possíveis vantagens que a utilização do tablet pode oferecer, por se tratar de uma tecnologia sem fio. Seguem as principais respostas:

Bom, eu acho que é muito bom, é como eu já falei no gerenciamento do meu tempo dentro da unidade... No tablet fica mais rápido até porque eu tenho a possibilidade de resgatar o que foi feito e ali alterar, para aumentar, diminuir. (E2)

Eu não fico limitada com ele móvel, me permite mobilidade. (E3) 
Na área de saúde eu acho fundamental, eu ganho tempo, gerencio melhor minhas ações de modo que é mais fácil manter algumas coisas, suspender alguns diagnósticos, lançar mão de outros me facilita muito... A ferramenta da tecnologia me ajuda muito. (E4)

As falas destacadas mostram que há consenso entre as enfermeiras quanto à importância da tecnologia móvel para registros. As vantagens mencionadas se referem, principalmente, a requisitos como mobilidade e agilidade para realizar a documentação de informações, otimizando assim o tempo destes profissionais, além da flexibilidade permitida na gerência das ações de cuidado com o uso do tablet.

\section{DISCUSSÃO}

Os participantes do estudo têm um tempo considerável de formação e de atuação na UTI Neonatal, o que sugere grande experiência e conhecimento nessa área, mas que também pode ser um indicativo de resistência à implantação de uma nova metodologia de documentação dos registros de enfermagem. Apenas uma das participantes tem pós-graduação específica na área em que atua e as demais não têm conhecimento específico no campo da informática que tenha sido adquirido por meio da realização de cursos ou capacitações.

As falas das enfermeiras enfatizam a ausência de recursos tecnológicos suficientes para o desenvolvimento das atividades de documentação dos profissionais de saúde da unidade, porém, isto não se trata apenas de uma realidade local. Em um estudo que avaliou um sistema desenvolvido para documentar o processo de enfermagem em um hospital de São Paulo, os relatos obtidos foram semelhantes aos das participantes deste estudo. Os entrevistados afirmaram que o computador é utilizado por outros profissionais, por isso os enfermeiros só podem usá-lo após a visita médica, e que o número de computadores não atende à demanda de uso do sistema, já que outros profissionais utilizam os equipamentos, o que atrasa o serviço e a utilização do programa ${ }^{(12)}$. Nesse sentido, os aplicativos para dispositivos móveis são uma excelente alternativa para resolver esta problemática.

Apesar de relatarem dificuldades em utilizar a tecnologia, as enfermeiras salientaram que elas foram superadas e que correspondem a problemas que são enfrentados inicialmente, na fase de adaptação 'ao novo' no processo de trabalho. Prova disso é que uma das participantes, em seus primeiros manuseios, apresentou grande dificuldade de utilizar o tablet, tendo em vista a sensibilidade da tela, mas, depois de algumas tentativas, evoluiu e manuseou o dispositivo sem mais dificuldades. Outro estudo realizado em Unidades Básicas de Saúde do município de Ribeirão Preto, estado de São Paulo, onde foi desenvolvido um software para dispositivos móveis, visando à educação permanente de profissionais da Enfermagem, contatou que $89 \%$ dos participantes também se consideravam iniciantes quanto ao nível de habilidade com as tecnologias computacionais e tinham dificuldade para o uso do tablet $^{(13)}$.

As profissionais que disseram não apresentar dificuldades afirmaram que têm uma tecnologia semelhante à do tablet, seja um dispositivo móvel do tipo smartphone ou uma tecnologia idêntica à utilizada no estudo. Essa familiaridade justifica a facilidade de utilização. Autores afirmam que a tecnologia móvel tornou-se onipresente. Smartphones e tablets são acessíveis e, consequentemente, mais fáceis de utilizar e populares entre profissionais da enfermagem. De acordo com pesquisa realizada com enfermeiros norte-americanos, dos 1.100 entrevistados, mais de $75 \%$ responderam que possuem um smartphone ou um tablet. Esta realidade representa uma tendência mundial ${ }^{(14)}$.

Das vantagens citadas pelas enfermeiras, destacou-se a mobilidade, ou seja, a "liberdade" que a tecnologia móvel permite no que se refere à locomoção do profissional. Também em estudo citado anteriormente, $90,9 \%$ dos profissionais de enfermagem que participaram da pesquisa fizeram uma avaliação positiva do uso de um aplicativo para dispositivo móvel (tablet). As razões para tal justificativa são a praticidade, a flexibilidade de horário, a facilidade de acessá-lo e de usá-lo em qualquer ambiente do local de trabalho e o fato de ser uma ferramenta que pode ser utilizada de diversas formas e para vários fins ${ }^{(13)}$.

Outros autores referem que os dispositivos móveis apresentam grandes vantagens, entre elas, 
a de ser portátil (capaz de ser transportado com relativa facilidade), utilizável e funcional, de fácil conectividade e comunicação com os usuários e com outros dispositivos. Outro aspecto importante é a facilidade de movimentação que o usuário pode ter, pois o dispositivo móvel cabe na palma da mão, melhora a qualidade visual e é mais confortável, leve, de baixo custo e discreto ${ }^{(15)}$.

Os profissionais da área de saúde têm uma quantidade cada vez maior de informações clínicas para gerenciar. Fazer isso, de forma eficaz e eficiente, é fundamental para o contexto atual dos serviços de saúde. As tecnologias da informação móveis, como os assistentes digitais pessoais (PDAs) e os computadores de mão (tablets), dão uma solução para gerenciar essas informações diretamente no ponto de atendimento. Essa é uma forma mais rápida e proativa de coletar dados mais fidedignos. Essas inovações podem superar desafios e fazer dos sistemas já existentes tecnologias mais eficientes, melhorar a usabilidade e salvar dados em apenas alguns segundos. Assim, tem-se assistência eficaz, quando estendida para os diversos aspectos do cuidado em saúde ${ }^{(16-17)}$.

Sabe-se que, na área de Enfermagem, os avanços da informática visam, primordialmente, aumentar o tempo disponível do profissional para as atividades relacionadas ao cuidado, proporcionando assistência mais humanizada, uma vez que, com o seu uso, as atividades burocráticas são mais ágeis, e esse tempo é revertido em assistência direta e eficaz aos pacientes. Os enfermeiros gastam cerca de um terço do seu tempo em atividades de localização, procura/busca, agregação e processamento de dados ou informações dos pacientes ${ }^{(18)}$. Desta maneira, os dispositivos móveis correspondem a uma estratégia que pode diminuir significativamente o tempo despedindo em atividades de documentação.

As documentações de enfermagem informatizadas reduzem o tempo despendido com o registro de informações, e sua utilização em dispositivos móveis facilita a entrada de dados com um toque no dispositivo, que diminui as digitadas por meio do teclado ${ }^{(19)}$.

Destaca-se ainda, a importância da inserção das tecnologias durante a graduação de enfermagem, para que, assim, os futuros profissionais desta área sejam adeptos do avanço tecnológico e dos benefícios que este propicia à assistência. Um estudo realizado com discentes de enfermagem da Universidade Federal do Ceará mostrou que, além do permitir a proximidade dos acadêmicos com as diferentes tecnologias, os suportes tecnológicos educacionais mostraram-se ferramenta válida, uma vez que foi possível identificar algumas particularidades e boa aceitação por parte dos futuros enfermeiros, sendo assim uma ferramenta complementar ao ensino presencial $^{(20)}$.

\section{CONCLUSÃO}

O mundo da informática passa por constantes transformações. Os recursos tecnológicos estão presentes na vida das pessoas, sendo utilizados desde os momentos de lazer até a realização de atividades profissionais. Nesse contexto, o advento das tecnologias móveis e sua crescente inserção no cotidiano de trabalho dos indivíduos exigem dos profissionais da saúde e da Enfermagem familiaridade com esses avanços, considerando os benefícios que podem ser adquiridos com sua utilização.

Foi constatado que a tecnologia móvel flexibiliza a realização das atividades de registro pelo enfermeiro, porque a coleta de dados pode ser realizada à beira do leito, ou seja, junto do paciente, e o processo de enfermagem pode ser desenvolvido em qualquer local da unidade, já que a tecnologia não depende de fios para que ele funcione.

Outra vantagem observada foi à otimização do tempo do enfermeiro, já que a informatização de atividades burocráticas e a mobilidade adquirida pelo uso do tablet resultam em mais eficácia e eficiência dos registros de enfermagem, possibilitando economia de tempo que pode ser revertida para o cuidado com o cliente e padronizar as evoluções, essenciais à continuidade da assistência ao cliente e à avaliação por outros profissionais.

Como limitações do estudo, destaca-se o tempo reduzido de manuseio do sistema para tecnologia móvel pelas enfermeiras, o número reduzido de participantes, bem como a utilização de um banco de dados que necessita de melhorias para se adequar à prática assistencial da UTIN. Além disso, o estudo mostra a realidade de apenas um setor do hospital escola. 
Conclui-se que as tecnologias da informação, associadas aos dispositivos móveis, contribuem sobremaneira com o processo de trabalho dos enfermeiros, razão por que é necessário investir mais em estudos que visem informatizar o processo de enfermagem e inserir novas tecnologias no cotidiano desses profissionais, não somente em UTIN, mas em todas as unidades e local de trabalho do enfermeiro.

\section{REFERÊNCIAS}

1. Cruz NS, Soares DKS, Bernardes A, Gabriel CS, Pereira MCA, Évora YDM. A competência técnica em informática de alunos de enfermagem. Rev. esc. enferm. USP. [Internet] 2011; 45(n.spe) [acesso em 14 nov 2014]. Disponível: http://dx.doi.org/10.1590/S0080-62342011000700009.

2. Sandi LB, Saccol AZ. Sobrecarga de informações geradas pela adoção de tecnologias da informação móveis e sem fio e suas decorrências para profissionais de vendas. RESI [Internet] 2010; 9(2) [acesso em 10 jun 2014]. Disponível: http://www.spell.org.br/documentos/ver/5491/sobrecarga-de-informacoes-geradas-pela adocaode-tecnologias-da-informacao-moveis-e-sem-fio-e-suas-decorrencias-para-profissionais-de-vendas.

3. Martha AS, Salomão PL, Romani R, Campos CJR, Sigulem D. Clinic web: PEP e interação com dispositivos móveis. ResearchGate. [Internet] 2014 [acesso em 10 jun 2014]. Disponível: http://www.researchgate.net/ publication/237565235_Clinic_Web_PEP_e_interao_com_dispositivos_mveis.

4. Sperandio DJ, Évora YDM. Nursing care planning: proposal for a software prototype. Rev. Latino-Am. Enfermagem. [Internet] 2005; 13(6) [acesso em 10 mai 2013]. Disponível: http://dx.doi.org/10.1590/S010411692005000600004.

5. Saccol A, Camarotto F. A adoção de tecnologias da informação móveis e sem fio (TIMS) e as competências de profissionais de vendas: dois estudos de caso na indústria farmacêutica de Goiás. Universitas Gestão e TI. [Internet] 2013; 3(1) [acesso em 13 jun 2013]. Disponível: https://dx.doi.org/10.5102/un.gti.v3i1.1959.

6. Oliveira APC, Coelho MEAA, Almeida VCF, Lisboa KWSC, Macêdo ALS. Sistematização da assistência de enfermagem: implementação em uma unidade de terapia intensiva. Rev. Rene. [Internet] 2012; 13(3) [acesso em 10 nov 2014]. Disponível: http://www.repositorio.ufc.br/ri/bitstream/riufc/4742/1/2012_art_vcfalmeida.pdf.

7. Menezes Junior JV, D'Castro RJ, Rodrigues FMM, Gusmão CMG, Lyra NRS, Sarinho SW. InteliMed: uma experiência de desenvolvimento de sistema móvel de suporte ao diagnóstico médico. RBCA. [Internet] 2011; 3(1) [acesso em 10 nov 2014]. Disponível: http://dx.doi.org/10.5335/rbca.2011.004.

8. Palomares MLE, Marques IR. Contribuições dos sistemas computacionais na implantação da sistematização da assistência de enfermagem. J. Health Inform. [Internet] 2010; 2(3) [acesso em 15 fev 2014]. Disponível: http:// www.jhi-sbis.saude.ws/ojs-jhi/index.php/jhi-sbis/article/view/94.

9. Tobase L, Guareschi APDF, Frias MAE, Prado C, Peres HHC. Recursos tecnológicos na educação em enfermagem. J. Health Inform. [Internet] 2013; 5(3) [acesso em 30 jan 2013]. Disponível: http://www.jhi-sbis.saude.ws/ojs-jhi/ index.php/jhi-sbis/article/view/218.

10. Bardin L. Análise de Conteúdo. Lisboa: Edições 70; 2009.

11. Ministério da Saúde (BR). Conselho Nacional de Saúde. Diretrizes e normas regulamentadoras de pesquisa envolvendo seres humanos. Resolução n. 466, de 12 de dezembro de 2012. Brasília; 2012.

12. Peres HHC, Lima AFC, Cruz DALM, Gaidzinski RR, Oliveira NB, Ortiz DCF, et al. Avaliação de sistema eletrônico para documentação clínica de enfermagem. Acta paul. enferm. [Internet] 2012; 25(4) [acesso em 28 mai 2014]. Disponível: http://dx.doi.org/10.1590/S0103-21002012000400010.

13. Tognoli SH. Medida indireta da pressão arterial: avaliação de programa de educação permanente oferecido em dispositivo móvel [dissertação]. Ribeirão Preto (SP): Universidade de São Paulo, Escola de Enfermagem de Ribeirão Preto; 2012.

14. Swan BA, Smith KA, Frisby A, Shaffer K, Hanson-Zalot M, Becker J. Evaluating tablet technology in an 
undergraduate nursing program. Nurs Educ Perspect. [Internet] 2013; 34(3) [acesso em 30 out 2014]. Disponível: http://go.galegroup.com/ps/i.o?id=GALE\%7CA335824632\&v=2.1\&u=capes\&it=r\&p=AONE\&sw=w\&asid=2c7a349 70a6456af85f9f3e3a6b26fab.

15. Lunardi GL, Dolci DB, Wendland J. Internet móvel nas organizações: fatores de adoção e impactos sobre o desempenho. Rev. Adm. Contemp. [Internet] 2013; 17(6) [acesso em 20 jan 2014]. Disponível: http://www.scielo. $\mathrm{br} / \mathrm{pdf} / \mathrm{rac} / \mathrm{v} 17 \mathrm{n} 6 / \mathrm{a} 04 \mathrm{v} 17 \mathrm{n} 6 . \mathrm{pdf}$.

16. Schuerenberg BK. Tablet PCs Heed Nurses' Needs: Nursing group upgrades to Tablet PCs to help improve care, documentation and communication. Health data management. [Internet] 2013; 64 [citado em 30 out 2014]. Disponível: https://www.mhealthevidence.org/content/tablet-pcs-heed-nurses-needs.

17. Parro MC, Évora YDM. Desenvolvimento de software para a organização da informação de um serviço de saúde ocupacional hospitalar. Rev. Eletr. Enf. [Internet] 2011; 13(3) [acesso em 04 nov 2014]. Disponível: https:// www.revistas.ufg.br/index.php?journal=fen\&page=article\&op=view\&path\%5B\%5D=11847.

18. Matsuda LM, Higarashi IH, Évora YDM, Bernardes A. Percepção de enfermeiros sobre o uso do computador no trabalho. Rev. bras. enf. [Internet] 2014; 67(6) [acesso em 04 nov 2014]. Disponível em: http://dx.doi. org/10.1590/0034-7167.2014670613.

19. Duffy M. Tablet technology for nurses. Am J Nurs. [Internet] 2012; 112(9) [acesso em 20 jan 2014]. Disponível: http://dx.doi.org/10.1097/01.NAJ.0000418927.60847.44.

20. Frota NM, Barros LM, Costa AFA, Santos ZMSA, Caetano JA. Hipermídia educacional sobre punção venosa periférica: perspectiva de acadêmicos de enfermagem. Cogitare Enferm. [Internet] 2014; 19(4) [acesso em 20 set 2015]. Disponível: http://dx.doi.org/10.5380/ce.v19i4.35384. 